
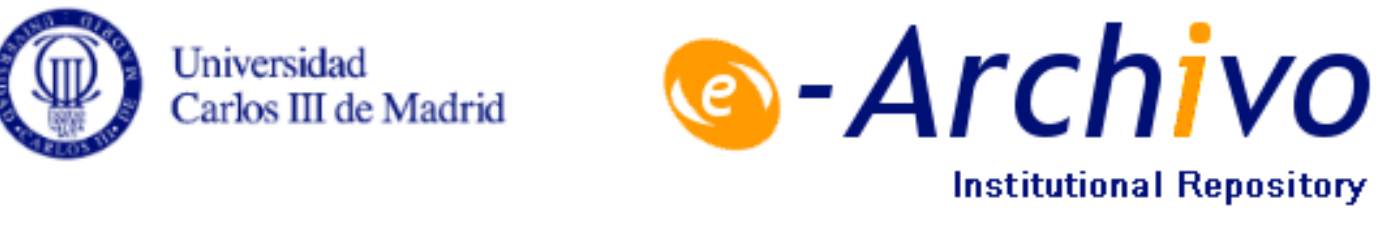

This document is published in:

IEEE Antennas and Wireless Propagation Letters 13 (2014) pp. 1309-1312

DOI: 10.1109/LAWP.2014.2336174

(C) 2014 IEEE. Personal use of this material is permitted. Permission from IEEE must be obtained for all other uses, in any current or future media, including reprinting/republishing this material for advertising or promotional purposes, creating new collective works, for resale or redistribution to servers or lists, or reuse of any copyrighted component of this work in other works. 


\title{
Combination of the Three Types of Diversity to Design High-Capacity Compact MIMO Terminals
}

\author{
M. Luz Pablo-González, Matilde Sánchez-Fernández, Senior Member, IEEE, and \\ Eva Rajo-Iglesias, Senior Member, IEEE
}

\begin{abstract}
Multiple-input-multiple-output (MIMO) schemes designed to increase channel capacity face strong limitations when a large number of antennas must be deployed in a terminal due to size constrains. This letter discusses how the different combinations of the three types of diversities - namely spatial, radiation pattern, and polarization diversity - can be used to get an improved capacity in compact terminals. The study compares realistic combinations that overcome the need for a large number of radiating elements in the user terminal.
\end{abstract}

Index Terms - Compact terminal, diversity, multiple-input-multiple-output (MIMO).

\section{INTRODUCTION}

I $\mathrm{N}$ THEIR initial conception, multiple-input-multipleoutput (MIMO) systems explode mainly spatial diversity to achieve an increase in channel capacity, that is, several radiating elements are separately placed. However, the limited size allowed at the terminal side and the need for large deployment of radiating elements [1] have motivated the use of the other types of diversities i.e., polarization diversity [2], [3] and radiation pattern diversity [4]-[6].

Many of the previous referred works are mixed strategies that simultaneously use two of the previously mentioned sources of diversity and have been shown to increase capacity or to overcome space limitation in the deployment of a large number of antennas. An example of that is multimode antennas that have been shown to be a good alternative in compact MIMO systems to provide the system with both spatial and radiation pattern diversity at the terminal side [6]. Multimode antennas, as the ones designed in [7], provide several radiation patterns, and the orthogonality or separability of these radiation patterns is key to provide full diversity. As the number of radiation patterns increases in a multimode antenna, the overlapping regions grow, and this increases correlation in the channel matrix, and therefore decreases spectral efficiency in most of channel knowledge and signal-to-noise ratio (SNR) scenarios. A way to overcome

This work was supported in part by the Spanish Ministry of Sci-ence and Innovation under Project GRE3NSYST (TEC2011-29006-C03-03), Project TEC2013-44019-R, and the CONSOLIDER-INGENIO 2010 program under Project COMONSENS (CSD 2008-00010).

The authors are with the Signal Theory and Communication Department, University Carlos III of Madrid, 28911 Leganés, Spain (e-mail: mluz@tsc.uc3m.es; mati@tsc.uc3m.es; eva@tsc.uc3m.es).

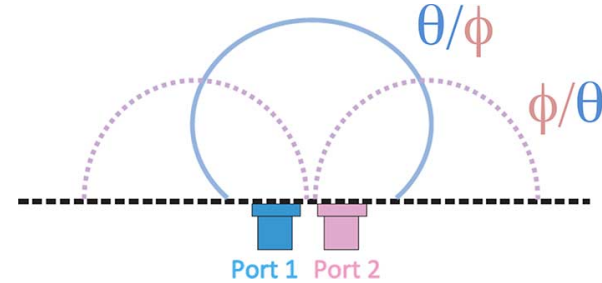

Fig. 1. Ideal antenna with radiation pattern and polarization diversity.

this problem is to add different orthogonal polarizations to each of the radiation patterns. This would not provide strictly a third source of diversity, given that each antenna only provides one polarization, but it will help to decrease the correlation in the radiation patterns.

As mentioned, the authors have proposed the use of radiation pattern diversity as a simple way of obtaining an increased channel capacity in [6] including the antenna design [7]. Now with this work, we propose to complete the study including also polarization as shown in Fig. 1. The objective is threefold: 1) to provide an additional source of diversity by itself; 2 ) to overcome modal correlation due to overlapping radiation patterns; and 3) to decrease the distance between antennas without significantly increasing channel correlation. Polarization diversity is well known as its use alone is previous to the development of MIMO systems, but even if we can find in the literature some examples of its combination with the other types of diversity [8]-[10], its aggregation has not been studied in a systematic way.

The purpose of the present work is to study the performance of the combination of the three types of diversities in the same terminal, enabling this way the design of compact terminals. A general channel model, extended from the one in [6], is provided, and the spectral efficiency study is made by simulation. We will show different scenarios combining two or three types of diversities, and we will derive general conclusions that can be applied afterwards to particular designs.

The letter is divided as follows. Section II details the developed channel model that has been used in the simulations and that includes the three diversities sources in the channel. Section III shows the most relevant results of the study of the combination of the diversities, and finally Section IV summarizes the main conclusions of the study.

\section{SOURCES OF DIVERSITY IN AN MIMO CHANNEL}

Multimode antennas are able to, at the same frequency, provide different field patterns, each of them with a separate feeding 
port. Several multimode antennas placed at different positions provide space diversity. Each multimode antenna may provide the system with two additional sources of diversity. The first one is the radiation pattern diversity; each mode of the antenna may provide different radiation patterns that are simultaneously active and covering different spatial ranges. Furthermore, each of these patterns may provide different polarizations, allowing the system with the second source of diversity.

Thus, for our model, the basic element in the antenna array is not the antenna anymore, but each of the field patterns (radiation patterns with its corresponding polarization), which we will identify as mode. The number of multimode antennas in the transmitter and receiver is respectively set to $M$ and $N$. For simplicity, we assume that all the antennas at the transmitter have the same number of modes $D_{\mathrm{t}}$ and for the receiver $D_{\mathrm{r}}$.

If we assume a flat fading characteristic for the channel, the MIMO channel is described by an $N D_{\mathrm{r}} \times M D_{\mathrm{t}}$ channel matrix $\mathbf{H}$, where $h_{n m}^{j i}$ describes the fading path from the $i$ th $(i=$ $\left.1 \ldots D_{\mathrm{t}}\right)$ port of the $m$ th $(m=1 \ldots M)$ transmitting antenna to the $j$ th $\left(j=1 \ldots D_{\mathrm{r}}\right)$ port of the $n$th receiving antenna $(n=1 \ldots N)$.

The fading coefficient $h_{n m}^{j i}$ can be modeled like the Green's function [11] sampled at the position of the $n$th receiving antenna $\mathbf{r}_{n}^{\prime}$ given that the $m$ th transmitting antenna is located at point source $\mathbf{r}_{m}$

$$
h_{n m}^{j i}=\int \mathbf{G}_{n(j)}^{\prime}\left(\mathbf{k}^{\prime}\right) \cdot \mathbf{E}_{\mathbf{r}}\left(\mathbf{k}^{\prime}, \mathbf{r}_{m}, i\right) e^{j \mathbf{k}^{\prime} \cdot \mathbf{r}_{n}^{\prime}} d \mathbf{k}^{\prime}
$$

where $\mathbf{G}_{n(j)}^{\prime}\left(\mathbf{k}^{\prime}\right)$ is the field pattern of $j$ th mode in receive antenna $n$ in $\mathbf{k}^{\prime}$ vector direction and $\mathbf{E}_{\mathbf{r}}\left(\mathbf{k}^{\prime}, \mathbf{r}_{m}, i\right)$ is the received electric field originated at the transmitted side by the $i$ th port of $m$ th antenna placed at $\mathbf{r}_{m}$. It should be noted that the field pattern carries information on the polarization and radiation pattern of the multimode antenna. Respectively, the received electric field can be modeled as the superposition of the scattered transmitted field $\mathbf{E}_{\mathbf{t}}\left(\mathbf{k}, \mathbf{r}_{m}, i\right)$

$$
\begin{aligned}
\mathbf{E}_{\mathbf{r}}\left(\mathbf{k}^{\prime}, \mathbf{r}_{m}, i\right) & =\int \mathbf{S}\left(\mathbf{k}^{\prime}, \mathbf{k}\right) \cdot \mathbf{E}_{\mathrm{t}}\left(\mathbf{k}, \mathbf{r}_{m}, i\right) d \mathbf{k} \\
& =\int \mathbf{S}\left(\mathbf{k}^{\prime}, \mathbf{k}\right) \cdot \mathbf{G}_{m(i)}(\mathbf{k}) e^{-j \mathbf{k} \cdot \mathbf{r}_{m}} d \mathbf{k}
\end{aligned}
$$

where $\mathbf{S}\left(\mathbf{k}^{\prime}, \mathbf{k}\right)$ is the transformation dyad that randomly changes the direction of the transmitted field [12]. The second equality comes from the fact that the transmitted field is a superposition of the plane waves $\mathbf{k}$, originated by the antenna in position $\mathbf{r}_{m}$ with a field pattern $\mathbf{G}_{m(i)}(\mathbf{k})$.

The vector space can be sampled into $L$ plane waves in the transmitter $\mathbf{k}_{\mathrm{t}}$ with $t=1 \ldots L$ and $L^{\prime}$ plane waves in the receiver $\mathbf{k}_{\mathrm{r}}^{\prime}$ with $r=1 \ldots L^{\prime}$ to cover all the space. Then, from (1) and (2)

$$
h_{n m}^{j i}=\sum_{\mathrm{r}, \mathrm{t}} \mathbf{G}_{n(j)}^{\prime}\left(\mathbf{k}_{\mathrm{r}}^{\prime}\right) \cdot \mathbf{S}\left(\mathbf{k}_{\mathrm{r}}^{\prime}, \mathbf{k}_{\mathrm{t}}\right) \cdot \mathbf{G}_{m(i)}\left(\mathbf{k}_{\mathrm{t}}\right) e^{-j \mathbf{k}_{\mathrm{t}} \cdot \mathbf{r}_{m}} e^{j \mathbf{k}_{\mathbf{r}}^{\prime} \cdot \mathbf{r}_{n}^{\prime}} .
$$

\section{A. Diversity Scenarios}

Each multimode antenna field pattern may provide the system with two sources of diversity, radiation pattern and polarization diversity, since each multimode field pattern provides a particular radiation pattern with a specific polarization in the direction of maximum radiation. In the general model provided in (3), polarization diversity maybe fully introduced to provide each radiation pattern with the two polarizations, to prevent the overlapping areas of the radiation patterns that generate high correlation [6] or to decrease the distance between the multimode antennas.

We next present all combinations of radiation pattern-polarization-spatial diversity that arise from different particularizations of the channel model in (3). For the sake of simplicity, we focus on the diversity scenarios at the terminal side; all diversity scenarios provided next could have a straightforward extension to the transmitter side.

- Scenario I (radiation pattern/spatial diversity): The number of antennas is $N>1$. The number of modes is $D_{\mathrm{r}}>1$, thus the field pattern $\mathbf{G}_{n(j)}^{\prime}\left(\mathbf{k}^{\prime}\right)$ has a different radiation pattern $\mathcal{G}_{n(j)}^{\prime}\left(\mathbf{k}^{\prime}\right)$ for each of the modes, and all the modes have the same polarization. This scenario is the one presented in [6].

- Scenario II (polarization/spatial diversity): The number of antennas is $N>1$. The number of modes is $D_{\mathrm{r}}=2$ if we assume two orthogonal polarizations in each of the antennas, and thus the field patterns $\mathbf{G}_{n(1)}^{\prime}\left(\mathbf{k}^{\prime}\right)$ and $\mathbf{G}_{n(2)}^{\prime}\left(\mathbf{k}^{\prime}\right)$ have a common overlapping radiation pattern $\mathcal{G}_{n}^{\prime}\left(\mathbf{k}^{\prime}\right)$ and the two mentioned orthogonal polarizations.

- Scenario III (polarization/radiation pattern): The number of antennas is $N=1$. The number of modes is given by the number of different radiation patterns and polarization scenarios. Assuming two different radiation patterns $\mathcal{G}_{n(1)}^{\prime}\left(\mathbf{k}^{\prime}\right)$ and $\mathcal{G}_{n(2)}^{\prime}\left(\mathbf{k}^{\prime}\right)$ and each of them has two polarizations, then $D_{\mathrm{r}}=4$.

- Scenario IV (polarization/radiation pattern/spatial diversity): This scenario has two different implementations, in both cases with $N>1$. If we restrict the number of radiation patterns to two $\mathcal{G}_{n(1)}^{\prime}\left(\mathbf{k}^{\prime}\right)$ and $\mathcal{G}_{n(2)}^{\prime}\left(\mathbf{k}^{\prime}\right)$, we can have just one polarization in each of them, thus $D_{\mathrm{r}}=2$ and $\mathbf{G}_{n(1)}^{\prime}\left(\mathbf{k}^{\prime}\right)$ and $\mathbf{G}_{n(2)}^{\prime}\left(\mathbf{k}^{\prime}\right)$ are orthogonal (Scenario IV.a). Also, we could implement both polarizations in each of the radiation patterns, and then $D_{\mathrm{r}}=4$ and $\mathbf{G}_{n(1)}^{\prime}\left(\mathbf{k}^{\prime}\right)$ and $\mathbf{G}_{n(2)}^{\prime}\left(\mathbf{k}^{\prime}\right)$ would have the same polarization and $\mathbf{G}_{n(3)}^{\prime}\left(\mathbf{k}^{\prime}\right)$ and $\mathbf{G}_{n(4)}^{\prime}\left(\mathbf{k}^{\prime}\right)$ would also have the same polarization orthogonal to the one in $\mathbf{G}_{n(1)}^{\prime}\left(\mathbf{k}^{\prime}\right)$ and $\mathbf{G}_{n(2)}^{\prime}\left(\mathbf{k}^{\prime}\right)$ (Scenario IV.b). The first scenario is the one improving radiation pattern correlation by means of polarization.

To characterize the transformation dyad $\mathbf{S}\left(\mathbf{k}_{\mathrm{r}}^{\prime}, \mathbf{k}_{\mathrm{t}}\right)$ in (3) for any of the scenarios, we assume the channel entries Gaussian and that the scatterers are independent and randomly change the plane wave impinging in direction $\mathbf{k}_{\mathrm{t}}$ to $\mathbf{k}_{\mathrm{r}}^{\prime}$. Furthermore, we assume a separable model for the joint power angular spectrum (PAS) $\mathcal{P}\left(\mathbf{k}_{\mathrm{r}}^{\prime}, \mathbf{k}_{\mathrm{t}}\right)=\mathcal{P}^{\prime}\left(\mathbf{k}_{\mathrm{r}}^{\prime}\right) \mathcal{P}\left(\mathbf{k}_{\mathrm{t}}\right)$ [13] and the angle between the field pattern in the transmitter/receiver and the electric field scattered noted as $\delta_{\mathrm{t}}$ and $\delta_{\mathrm{r}}^{\prime}$, respectively.

At the bottom of the next page, we present the formulation for $h_{n m}^{j i}$ for each of the diversity scenarios presented assuming the same diversity scenario in both the transmitter and the receiver. $g_{\mathrm{rt}}$ is a zero-mean complex Gaussian random variable with unit variance. 
It should be noted here that mutual coupling between modes or antenna elements is not considered in our proposed model. However, it could be easily included by means of a coupling matrix $\mathbf{C}$ as shown in [14], given the separable matrix model that arise in the definition of $\mathbf{H}$ as a product of several matrices for any of the diversity scenarios defined.

\section{SPeCtRal EfFiciency STUdy}

Focusing in the downlink, any simulation study regarding channel capacity needs different realizations of $\mathbf{H}$, and this implies a particular sampling $\mathbf{k}_{\mathrm{r}}^{\prime}$ and $\mathbf{k}_{\mathrm{t}}$ of the wave space defined by the space vectors $\mathbf{k}^{\prime}$ and $\mathbf{k}$ and a particular antenna geometry. As a first approach, and given that the elevational angle spread is being measured to be much less than the azimuthal spread [15], for all the simulations provided here, just azimuthal angle is taken into account, and it will be sampled uniformly $\phi \in(0,2 \pi)$ generating $L=L^{\prime}=900$. Also, linear arrays are considered with antenna separation of $d_{\mathrm{t}}=d_{\mathrm{r}}=0.5 \lambda$, except when other separation is explicitly noted. Other $d_{\mathrm{t}}$ and $d_{\mathrm{r}}$ have also been tested without significant change in the relative behavior. Simulation results evaluate the spectral efficiency with

$$
C=E\left\{\log \operatorname{det}\left(\mathbf{I}_{N D_{\mathrm{r}}}+\frac{\mathrm{SNR}}{M D_{\mathrm{r}}} \mathbf{H H}^{\dagger}\right)\right\} .
$$

Simulations are made by generating 5000 independent samples of $\mathbf{H}$ matrix and averaging them to get the achievable rate.

With the aim of designing compact terminals, the results show the performance of the different diversity scenarios presented in Section II providing this diversity only at the receiver (terminal) side. It should be noted then that at the transmitter side, a fixed number $(M=4)$ of omnidirectional antennas $\left(D_{\mathrm{t}}=1\right)$ is used. In some of the figures, we also use two reference scenarios for the receiver side, which are the classical MIMO scheme with $N=4$ omnidirectional antennas $\left(D_{\mathrm{r}}=1\right)$ and the same scheme but with $N-1$ antennas.

Initially, we assume we can have antennas with two ports, each port with the same broadside radiation pattern, but each one of them with a different orthogonal polarization (Scenario II). These antennas can be easily implemented as combinations of dipoles and shaped ring slots [8]. To further reduce the total antenna size, we also propose to use a single multimode antenna with four ports providing two different radiation patterns with the two polarizations each (Scenario III) [16]. These results are

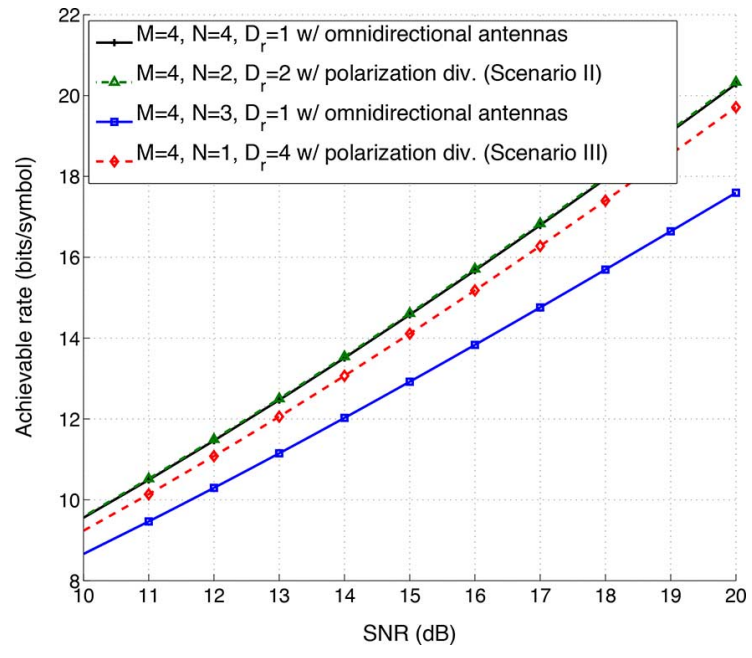

Fig. 2. Spectral efficiency with multimode antennas with polarization diversity.

included in Fig. 2. We can observe for the two proposals that we get similar performance to the reference scenario with four omnidirectional antennas and an important increase with respect to the three antennas with a significant decrease of the antenna size as we have only $N=2$ or $N=1$ antennas.

Next, we fully introduce pattern diversity with each port of the antenna radiating with a different radiation pattern. These results are presented in Fig. 3. We compare the case where the two radiation patterns have the same polarization (Scenario I) to a case where they have orthogonal polarizations (Scenario IV.a). The latter clearly overcomes correlation due to the radiation pattern overlapping and represents a case where the three diversities are used simultaneously. Furthermore, in this scheme, the performance approaches the one of four antennas in a row with the same interelement distance reducing again the size of the terminal. When simplicity is a requirement, the use of multimode antennas can be seen as a drawback. In this scenario, we can still design compact terminals not by reducing the number of radiating elements, but by decreasing the distance between elements. With this purpose, we have now studied whether the use of simple antennas with different polarizations can bring any advantage. Particularly, we propose to alternate two orthogonal polarizations for the antennas, but to keep the same radiation pattern for all of them, and all of them are also simple single-port
Scenario I

Scenario II

Scenarios III and IV.b

Scenario IV.a $\left.h_{n m}^{j i}=\sum_{\mathrm{r}, \mathrm{t}} \mathcal{G}_{n(j)}^{\prime}\left(\mathbf{k}_{\mathrm{r}}^{\prime}\right) \mathcal{P}^{\prime}\left(\mathbf{k}_{\mathrm{r}}^{\prime}\right) g_{\mathrm{rt}} \mathcal{P}\left(\mathbf{k}_{\mathrm{t}}\right) \mathcal{G}_{m(i)}\left(\mathbf{k}_{\mathrm{t}}\right)\right) e^{-j \mathbf{k}_{\mathrm{t}} \cdot \mathbf{r}_{m}} e^{j \mathbf{k}_{\mathrm{r}}^{\prime} \cdot \mathbf{r}_{n}^{\prime}}$

$h_{n m}^{11}=\sum_{\mathrm{r}, \mathrm{t}} \mathcal{G}_{n}^{\prime}\left(\mathbf{k}_{\mathrm{r}}^{\prime}\right) \cos \left(\delta_{\mathrm{r}}^{\prime}\right) \mathcal{P}^{\prime}\left(\mathbf{k}_{\mathrm{r}}^{\prime}\right) g_{\mathrm{rt}} \mathcal{P}\left(\mathbf{k}_{\mathrm{t}}\right) \cos \left(\delta_{\mathrm{t}}\right) \mathcal{G}_{m}\left(\mathbf{k}_{\mathrm{t}}\right) e^{-j \mathbf{k}_{\mathrm{t}} \cdot \mathbf{r}_{m}} e^{j \mathbf{k}_{\mathrm{r}}^{\prime} \cdot \mathbf{r}_{n}^{\prime}}$

$h_{n m}^{22}=\sum_{\mathrm{r}, \mathrm{t}} \mathcal{G}_{n}^{\prime}\left(\mathbf{k}_{\mathrm{r}}^{\prime}\right) \sin \left(\delta_{\mathrm{r}}^{\prime}\right) \mathcal{P}^{\prime}\left(\mathbf{k}_{\mathrm{r}}^{\prime}\right) g_{\mathrm{rt}} \mathcal{P}\left(\mathbf{k}_{\mathrm{t}}\right) \sin \left(\delta_{\mathrm{t}}\right) \mathcal{G}_{m}\left(\mathbf{k}_{\mathrm{t}}\right) e^{-j \mathbf{k}_{\mathrm{t}} \cdot \mathbf{r}_{m}} e^{j \mathbf{k}_{\mathrm{r}}^{\prime} \cdot \mathbf{r}_{n}^{\prime}}$

$h_{n m}^{11}=\sum_{\mathrm{r}, \mathrm{t}} \mathcal{G}_{n(1)}^{\prime}\left(\mathbf{k}_{\mathrm{r}}^{\prime}\right) \cos \left(\delta_{\mathrm{r}}^{\prime}\right) \mathcal{P}^{\prime}\left(\mathbf{k}_{\mathrm{r}}^{\prime}\right) g_{\mathrm{rt}} \mathcal{P}\left(\mathbf{k}_{\mathrm{t}}\right) \cos \left(\delta_{\mathrm{t}}\right) \mathcal{G}_{m(1)}\left(\mathbf{k}_{\mathrm{t}}\right) e^{-j \mathbf{k}_{\mathrm{t}} \cdot \mathbf{r}_{m}} e^{j \mathbf{k}_{\mathrm{r}}^{\prime} \cdot \mathbf{r}_{n}^{\prime}}$

$h_{n m}^{22}=\sum_{\mathrm{r}, \mathrm{t}} \mathcal{G}_{n(2)}^{\prime}\left(\mathbf{k}_{\mathrm{r}}^{\prime}\right) \cos \left(\delta_{\mathrm{r}}^{\prime}\right) \mathcal{P}^{\prime}\left(\mathbf{k}_{\mathrm{r}}^{\prime}\right) g_{\mathrm{rt}} \mathcal{P}\left(\mathbf{k}_{\mathrm{t}}\right) \cos \left(\delta_{\mathrm{t}}\right) \mathcal{G}_{m(2)}\left(\mathbf{k}_{\mathrm{t}}\right) e^{-j \mathbf{k}_{\mathrm{t}} \cdot \mathbf{r}_{m}} e^{j \mathbf{k}_{\mathrm{r}}^{\prime} \cdot \mathbf{r}_{n}^{\prime}}$

$h_{n m}^{33}=\sum_{\mathrm{r}, \mathrm{t}} \mathcal{G}_{n(1)}^{\prime}\left(\mathbf{k}_{\mathrm{r}}^{\prime}\right) \sin \left(\delta_{\mathrm{r}}^{\prime}\right) \mathcal{P}^{\prime}\left(\mathbf{k}_{\mathrm{r}}^{\prime}\right) g_{\mathrm{rt}} \mathcal{P}\left(\mathbf{k}_{\mathrm{t}}\right) \sin \left(\delta_{\mathrm{t}}\right) \mathcal{G}_{m(1)}\left(\mathbf{k}_{\mathrm{t}}\right) e^{-j \mathbf{k}_{\mathrm{t}} \cdot \mathbf{r}_{m}} e^{j \mathbf{k}_{\mathrm{r}}^{\prime} \cdot \mathbf{r}_{n}^{\prime}}$

$h_{n m}^{44}=\sum_{\mathrm{r}, \mathrm{t}} \mathcal{G}_{n(2)}^{\prime}\left(\mathbf{k}_{\mathrm{r}}^{\prime}\right) \sin \left(\delta_{\mathrm{r}}^{\prime}\right) \mathcal{P}^{\prime}\left(\mathbf{k}_{\mathrm{r}}^{\prime}\right) g_{r t} \mathcal{P}\left(\mathbf{k}_{\mathrm{t}}\right) \sin \left(\delta_{\mathrm{t}}\right) \mathcal{G}_{m(2)}\left(\mathbf{k}_{\mathrm{t}}\right) e^{-j \mathbf{k}_{\mathrm{t}} \cdot \mathbf{r}_{m}} e^{j \mathbf{k}_{\mathrm{r}}^{\prime} \cdot \mathbf{r}_{n}^{\prime}}$

$h_{n m}^{11}=\sum_{\mathrm{r}, \mathrm{t}} \mathcal{G}_{n(1)}^{\prime}\left(\mathbf{k}_{\mathrm{r}}^{\prime}\right) \cos \left(\delta_{\mathrm{r}}^{\prime}\right) \mathcal{P}^{\prime}\left(\mathbf{k}_{\mathrm{r}}^{\prime}\right) g_{\mathrm{rt}} \mathcal{P}\left(\mathbf{k}_{\mathrm{t}}\right) \cos \left(\delta_{\mathrm{t}}\right) \mathcal{G}_{m(1)}\left(\mathbf{k}_{\mathrm{t}}\right) e^{-j \mathbf{k}_{\mathrm{t}} \cdot \mathbf{r}_{m}} e^{j \mathbf{k}_{\mathrm{r}}^{\prime} \cdot \mathbf{r}_{n}^{\prime}}$

$h_{n m}^{22}=\sum_{\mathrm{r}, \mathrm{t}} \mathcal{G}_{n(2)}^{\prime}\left(\mathbf{k}_{\mathrm{r}}^{\prime}\right) \sin \left(\delta_{\mathrm{r}}^{\prime}\right) \mathcal{P}^{\prime}\left(\mathbf{k}_{\mathrm{r}}^{\prime}\right) g_{\mathrm{rt}} \mathcal{P}\left(\mathbf{k}_{\mathrm{t}}\right) \sin \left(\delta_{\mathrm{t}}\right) \mathcal{G}_{m(2)}\left(\mathbf{k}_{\mathrm{t}}\right) e^{-j \mathbf{k}_{\mathrm{t}} \cdot \mathbf{r}_{m}} e^{j \mathbf{k}_{\mathrm{r}}^{\prime} \cdot \mathbf{r}_{n}^{\prime}}$ 


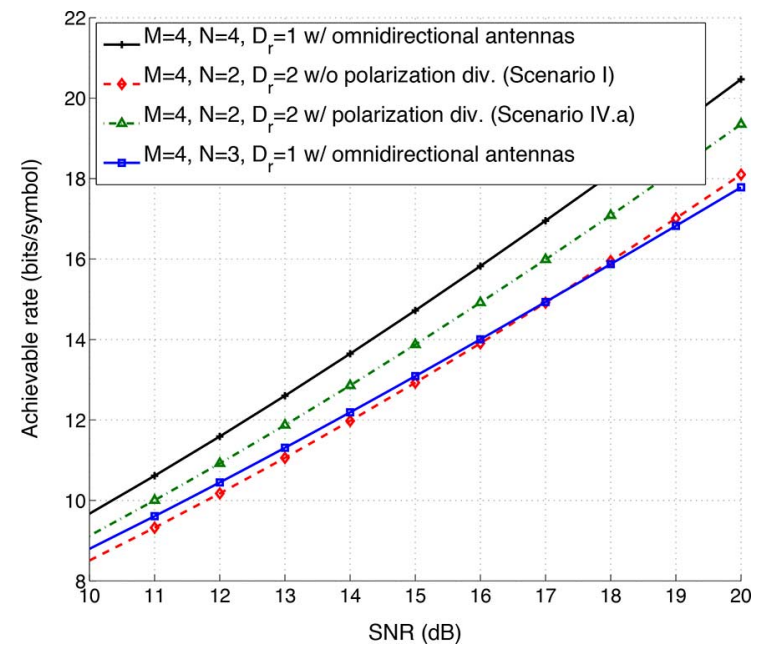

Fig. 3. Spectral efficiency with multimode antennas with pattern diversity.

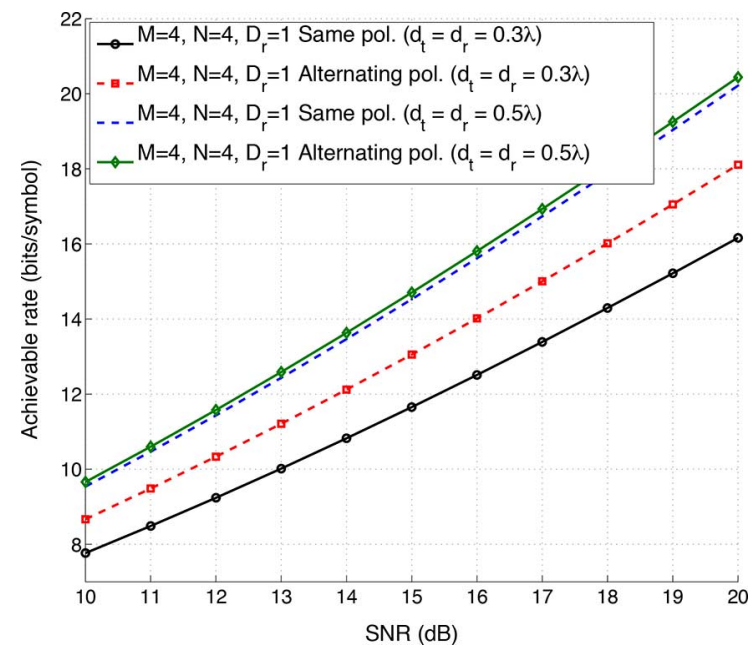

Fig. 4. Spectral efficiency when using identical monomode elements with same radiation pattern and alternating polarization for different interelement distances.

antennas. For this study, we have worked with broadside radiation pattern, and we have studied two cases with two interelement distances $d_{\mathrm{t}}=d_{\mathrm{r}}=0.3 \lambda$ and $d_{\mathrm{t}}=d_{\mathrm{r}}=0.5 \lambda$. The results are presented in Fig. 4 . We can conclude that we can significantly reduce the distance between elements, and by providing polarization diversity, we achieve higher capacity. For a distance of $d_{\mathrm{t}}=d_{\mathrm{r}} \geq 0.5 \lambda$, the use or not of polarization diversity does not give any advantage. In this particular case, despite the small distance between antennas, mutual coupling is not an issue given that alternate polarizations provide very low mutual coupling.

\section{DISCUSSION}

The introduction of more than one type of diversity allows the design of more compact terminals, given that the number of radiating elements needed is significantly decreased. The results presented in this study can serve as guidelines for terminal designers. Antennas providing combinations of the different types of diversities already exist in the literature. For instance, we can find compact designs for antennas with polarization diversity using the same radiation pattern as in [8], but also with different radiation patterns for each polarization [17]. Both cases refer to two-port antennas, but also a four-port antenna corresponding to two radiation patterns, each of them with two polarizations, can be implemented if desired as in [16], but assuming a more complicated and bigger antenna.

\section{REFERENCES}

[1] F. Rusek et al., "Scaling up MIMO: Opportunities and challenges with very large arrays," IEEE Signal Process. Mag., vol. 30, no. 1, pp. 40-60, Jan. 2013.

[2] J. Perez, J. Ibanez, L. Vielva, and I. Santamaria, “Approximate closed-form expression for the ergodic capacity of polarisation-diversity MIMO systems," Electron. Lett., vol. 40, no. 19, pp. 1192-1194, 2004.

[3] L. Dong, H. Choo, R. H. , Jr., and H. Ling, "Simulation of MIMO channel capacity with antenna polarization diversity," IEEE Trans. Wireless Commun., vol. 4, no. 4, pp. 1869-1873, Jul. 2005.

[4] C. Dietrich, K. Dietze, J. Nealy, and W. Stutzman, "Spatial, polarization, and pattern diversity for wireless handheld terminals," IEEE Trans. Antennas Propag., vol. 49, no. 9, pp. 1271-1281, Sep. 2001.

[5] T. Svantesson, "Correlation and channel capacity of MIMO systems employing multimode antennas," IEEE Trans. Veh. Technol., vol. 51, no. 6, pp. 1304-1312, Nov. 2002.

[6] M. Sánchez-Fernández, E. Rajo-Iglesias, O. Quevedo-Teruel, and M. Pablo-González, "Spectral efficiency in MIMO systems using space and pattern diversities under compactness constraints," IEEE Trans. Veh. Technol., vol. 57, no. 3, pp. 1637-1645, May 2008.

[7] E. Rajo-Iglesias, O. Quevedo-Teruel, and M. Sánchez-Fernández, "Compact multimode patch antennas for MIMO applications," IEEE Antennas Propag. Mag., vol. 50, no. 2, pp. 197-205, Apr. 2008.

[8] H. Li, J. Xiong, Z. Ying, and S. He, "Compact and low profile colocated MIMO antenna structure with polarisation diversity and high port isolation," Electron. Lett., vol. 46, no. 2, pp. 108-110, 2010.

[9] D. Piazza, P. Mookiah, M. D’Amico, and K. Dandekar, "Experimental analysis of pattern and polarization reconfigurable circular patch antennas for MIMO systems," IEEE Trans. Veh. Technol., vol. 59, no. 5, pp. 2352-2362, Jun. 2010.

[10] H. Chattha, Y. Huang, S. Boyes, and X. Zhu, "Polarization and pattern diversity-based dual-feed planar inverted-F antenna," IEEE Trans. Antennas Propag., vol. 60, no. 3, pp. 1532-1539, Mar. 2012.

[11] D. Chizhik, "Slowing the time-fluctuating MIMO channel by beam forming," IEEE Trans. Wireless Commun., vol. 3, no. 5, pp. 1554-1565, Sep. 2004.

[12] T. Svantesson, "A physical MIMO radio channel model for multielement multi-polarized antenna systems," in Proc. IEEE Veh. Technol. Conf., Oct. 2001, vol. 2, pp. 1083-1087.

[13] H. Xu, D. Chizhik, H. Huang, and R. Valenzuela, "A generalized spacetime multiple-input multiple-output (MIMO) channel model," IEEE Trans. Wireless Commun., vol. 3, no. 3, pp. 966-975, May 2004.

[14] P. Fletcher, M. Dean, and A. Nix, "Mutual coupling in multi-element array antennas and its influence on MIMO channel capacity," Electron. Lett., vol. 39, no. 4, pp. 342-344, Feb. 2003.

[15] H. Xu et al., "MIMO channel capacity for fixed wireless: measurements and models," in Proc. IEEE Veh. Technol. Conf., Oct. 2001, vol. 2, pp. 1068-1072.

[16] S.-L. Yang, K.-M. Luk, H.-W. Lai, A. Kishk, and K.-F. Lee, "A dualpolarized antenna with pattern diversity," IEEE Antennas Propag. Mag., vol. 50, no. 6, pp. 71-79, Dec. 2008.

[17] L. Zou and C. Fumeaux, "A cross-shaped dielectric resonator antenna for multifunction and polarization diversity applications," IEEE Antennas Wireless Propag. Lett., vol. 10, pp. 742-745, 2011. 